\title{
MIRANDO ENTRE LOS INTERSTICIOS DE LA BRECHA DIGITAL EN LA EDUCACIÓN SUPERIOR
}

\section{Introducción}

Entre 1983 y 2006 los inscritos en instituciones universitarias en Chile se multiplicaron por cuatro y para el 2012 se proyecta que un $50 \%$ de jóvenes de una misma cohorte ingresará a la educación superior. La sola ampliación de la cobertura y el ingreso de públicos forzosamente más diversos impone múltiples desafíos para todas las instituciones del sistema. Supone la revisión y el rediseño de muchos de los dispositivos y métodos pedagógicos actualmente en uso.

La Universidad de Chile comenzó en 1999 una reforma de los estudios de pregrado orientada a la superación de los paradigmas, tanto enciclopédico como profesionalizante, buscando mayor interdisciplinariedad y formación de personas reflexivas, críticas y creativas. Se pretende responder a las necesidades de una vida social y económica cada vez más dinámica, asumiendo que el estudiante es el protagonista de la construcción de sus propios conocimientos y del desarrollo de sus competencias.

Uno de los aspectos importantes de esta reforma ha sido la instauración en el 2000 de un dispositivo común a toda la universidad, consistente en un "pool" de cursos de formación general (CFG) bajo la inspiración, objetivos y conceptos de la reforma, y que se ofrece cada semestre a todos los estudiantes. Dichos cursos consideran, como una de sus innovaciones pedagógicas principales, la utilización de NTIC por la vía de la plataforma web http://www.plataforma.uchile.cl/fg/ home_cfg.htm. Se pretende así modernizar el proceso de aprendizaje, mejorando su calidad y garantizando su equidad.

En un plano mayor, las experiencias de evaluación en el campo de la docencia universitaria no son necesariamente habituales en 
Chile. A diferencia de países industrializados de América del Norte o Europa, los aspectos tocantes a la pedagogía universitaria no se encuentran suficientemente extendidos y/o aceptados como sujeto de investigación y de políticas específicas. Por otra parte, poco se sabe de los impactos netos de la incorporación de NTIC a los procesos de enseñanza y a la vida de las instituciones mismas: ¿cómo se distribuyen las asignaciones de equipamiento? ¿Cómo se construyen y orientan los dispositivos en dicho marco? ¿Qué ventajas y desventajas enfrentan y/o crean para los estudiantes y para los procesos de enseñanzaaprendizaje?, por ejemplo.

El presente artículo se propone analizar empíricamente algunos aspectos de la incorporación de NTIC a la enseñanza universitaria desde la perspectiva del público receptor y de los eventuales signos de la llamada "brecha digital" que éste pudiera evidenciar ${ }^{1}$. En particular, se intentará determinar cuáles son las condiciones de acceso y los comportamientos y capacidades de uso respecto de las NTIC, más allá (o ex-ante) de lo que toca directamente al proceso enseñanzaaprendizaje propuesto por esta universidad.

\section{El dispositivo estudiado y los datos utilizados}

En el primer semestre de 2004 el dispositivo CFG acogió 2.775 inscripciones equivalentes a 2.481 individuos, provenientes de 14 facultades, dos institutos y del programa de Bachillerato. Ello corresponde al $11 \%$ del total de estudiantes de pregrado para ese año, concentrados mayoritariamente en los primeros cuatro años de cada carrera (27\% en primer año, $37 \%$ en segundo y $36 \%$ en tercero o más) y con un promedio de edad de 21,8 años. Globalmente, el dispositivo y la muestra que de él fue extraída son representativos

1 Nos remitiremos aquí a la definición funcional dada por la Organización para la Cooperación y el Desarrollo Económico (OCDE, 2001) que comprende "brecha digital" como aquella existente entre individuos, hogares, empresas y áreas geográficas, de diferentes niveles socioeconómicos, en relación con las oportunidades de acceso a las TIC y al uso de éstas para una amplia variedad de actividades (Soupizet, 2003). Para un debate más profundo ver por ejemplo Raad (2006) o Araya (2005). 
del conjunto de la universidad, aunque con sesgos tales como una sobrerrepresentación femenina $(53,7 \%$ contra $46,4 \%)$ y de estudiantes de condición socioeconómica baja (36,4\% contra 31\%).

Al interior de la población del dispositivo se efectuaron dos cuestionarios con objetivos convergentes pero diferentes: uno al inicio y otro al final de los cursos. En total respondió un 47\% de estudiantes equivalentes a 1.288 inscripciones. El primer cuestionario, cuyos datos utilizaremos aquí, cuenta con 719 observaciones individuales y se orientó fundamentalmente a conocer las condiciones de trabajo y equipamiento de los estudiantes al momento de enfrentarse a los cursos inscritos. Estos datos constituyen la base del presente artículo y fueron complementados con otras bases (Programa de Atención Económica, PAE, y el Departamento de Evaluación, Medición y Registro Educacional, DEMRE). Por el contrario, el segundo cuestionario (829 observaciones) se orienta de lleno hacia las prácticas de estudio, uso y evaluación de los estudiantes respecto de tales cursos y sus sitios web una vez cursados.

La condición socioeconómica -que será nuestra variable referencia- se consideró de manera binaria a partir del trabajo que realiza el PAE. Dicho programa se basa en un minucioso trabajo de asistentes sociales y en una pauta de evaluación objetiva para calificar la situación de vulnerabilidad social y económica de los estudiantes ${ }^{2}$.

\section{Marco y estrategias de análisis}

En el estudio general realizado entre 2004 y 2005 se interrogó por las actitudes y usos concretos dados a las herramientas NTIC, en tanto susceptibles de expresarse como capital cultural en estado material y, a la vez, como capital cultural en estado incorporado (Bourdieu,

2 Entre los factores considerados por ese programa se cuentan, entre otros: ingreso per cápita $(<\$ 150.000)$, situación ocupacional, tenencia, tipo y avalúo de vivienda, instrucción del jefe de hogar. 
1979). Si el primero se relaciona con el acceso concreto y regular a ciertos bienes culturales tangibles, como libros, revistas, museos, entre otros, el segundo se refiere al dominio y a las disposiciones a su uso específico. En definitiva, se trata de un cierto saber "servirse de" y de la mayor o menor capacidad para extraer todo el valor de uso de tales bienes. En otras palabras, no basta sólo con tener "acceso a", es necesario además poseer y desarrollar ciertas disposiciones y habilidades para disfrutar de tales bienes.

Para el caso de las NTIC la interrogante resulta pertinente, pues se sabe que no basta con estar conectado a internet para ser usuario regular y, más aún, para obtener y/o amplificar sus beneficios. Según cifras del Programa de Naciones Unidas para el Desarrollo (PNUD), en 2001 se estimaba que un 33,4\% de personas poseía acceso a computadores, pero que sólo un 29,6\% se declaraba usuario habitual. Para el caso de internet, se constataba una situación similar, teniendo en cuenta que un $82,7 \%$ de los chilenos mayores de 18 años declaraba "no navegar" regularmente y que dos tercios de los usuarios de la web poseían entre seis y 29 años (PNUD, 2002). Según la Encuesta de Caracterización Económica (CASEN), en 2003 un $25 \%$ de los hogares tenía un computador y un 29,1\% de chilenos ya poseía acceso a internet. No obstante, el porcentaje de acceso en el quintil de mayores ingresos era cuatro veces superior al del quintil de menores ingresos, siendo los establecimientos educacionales un lugar preferencial de acceso ${ }^{3}$.

Evidentemente, se aprecia una proyección de la brecha social hacia el ámbito digital. Sin embargo, aparte de los económicos, otros factores han sido señalados como componentes y como creadores potenciales de brecha digital. En efecto, más allá del equipamiento tecnológico parecen existir barreras culturales, geográficas, de identidad, de edad, entre otras (Castellón y Jaramillo, 2001), y que dificultan la incorporación de las NTIC en la vida cotidiana de las

3 Otros estudios como el caso del World Internet Project Chile (WIP) (www.wipchile.cl) anotan cifras concordantes. 
personas, generando diferencias constitutivas de nuevas barreras sociales y culturales. La ausencia de las llamadas habilidades computacionales aparece además como una dificultad para la penetración de internet en diversos países, y otro tanto ocurriría con el interés y la experiencia (Herrera y Godoy, 2004), lo que parece ser un buen ejemplo de potenciales barreras acumulativas tocantes a capitales culturales incorporados.

Resulta pertinente interrogarse sobre el acceso, pero también respecto de la existencia de brechas que podrían ubicarse en lo que se conoce como segundo nivel de apropiación de las NTIC, referido a los saberes, trasfondos socioculturales y conocimientos que permiten usarlos en nuestra vida diaria (Araya, 2005). Es posible postular que las diferencias socioeconómicas, de género, y de capitales culturales y escolares ${ }^{4}$ entre estudiantes pueden ser fuente de origen para tales brechas, antes incluso, de enfrentarse a la experiencia de cursos asistidos mediante sitios web u otros.

Lo anterior presenta múltiples aristas, tocantes a la profundidad y orientación de las políticas públicas, al rol del sistema educacional y a la mutua determinación entre las dimensiones de calidad y equidad. Aunque Chile presenta indicadores y tendencias en torno a conectividad y equipamiento tecnológico que pueden ser vistas como alentadoras (Soupizet, 2003), dicho crecimiento resulta muy insuficiente (Brunner, 2001) y, más aún, se reparte de manera muy desigual.

Para el caso de la educación, la apropiación de las NTIC supone no sólo el acceso sino que, sobre todo, la capacidad de su uso y, por ende, un cierto capital cultural incorporado, susceptible

4 Por capital escolar deberá entenderse la presencia y acumulación de prácticas y técnicas de estudio. El promedio de las pruebas de Lenguaje y Matemáticas obtenido para ingresar a la universidad constituye un reflejo aceptable de dicho capital y será el indicador utilizado. Como las pruebas no son comparables de un año a otro, se construyó una variable ordinal basándose en el promedio y desviación estándar de los totales de quienes ingresaron a la Universidad de Chile entre 2000 y 2005. Para el capital cultural familiar se consideró la variable ordinal "número de libros en casa". 
de encontrarse desigualmente distribuido en la población estudiantil universitaria, a pesar de la homogeneidad que es posible suponer en instituciones académicamente selectivas.

Operacionalmente, y de acuerdo con nuestros datos, intentaremos comprender, situar y caracterizar la existencia de esta brecha digital en términos de capitales culturales materiales e incorporados, considerando como variables dependientes: los niveles de equipamiento, el tiempo de conexión declarado a internet, los tipos de usos preferentes dados a ella y el dominio de ciertos softwares ${ }^{5}$. Insistamos en que para dicho análisis no se considerará la experiencia misma de los CFG en donde los estudiantes fueron encuestados. En otras palabras, no se medirá aquí directamente el impacto del uso de NTIC para la enseñanza en el marco de ese programa (Roco, 2006) sino, más bien, las condiciones en que los estudiantes llegan a enfrentarse con éste.

\section{La distribución de capitales según condición socioeconómica}

Resumiendo las características de la muestra, los estudiantes utilizan el computador en promedio cinco días a la semana, dedicando cerca de un $52 \%$ del tiempo de uso a los estudios (todo tipo de cursos). Un $45 \%$ posee banda ancha mientras que un $25 \%$ no posee conexión propia a internet. Un $28 \%$ accede a la red en sus facultades y un $10 \%$ en casa de amigos, parientes o cybercafés ${ }^{6}$. Sólo un 0,8\% declara conectarse cero hora semanal, lo que se asimila a una frecuencia de uso esporádica. Un 63,6\% declara poseer un "dominio medio de internet" contra un $17,7 \%$ en "dominio alto". Finalmente, un 54\% posee grabador de CD y un $79 \%$ impresora al inicio de los CFG.

5 Para cada una de las cuales se testeará la independencia estadística respecto de otras variables. Nota: análisis hechos utilizando el programa SASß v.9.1

6 El "acceso prioritario" corresponde al espacio en el cual los estudiantes declaran conectarse a internet de manera preferente, pues las personas utilizan diferentes lugares (WIP, 2003) lo que es coherente con la situación propia de los estudiantes en términos de uso del tiempo y movilidad. 
Mientras el 88\% de estudiantes de la muestra declara poseer un computador, sólo un $77 \%$ de becados PAE se encuentra en dicha situación. Comparados con el 95\% de no becados que menciona poseer uno, la diferencia es significativa. Adicionalmente, sólo un $1,3 \%$ de la muestra afirma poseer un computador portátil a la fecha, y existen diferencias importantes entre no becados y becados respecto de la antigüedad de los sistemas operativos de sus respectivos computadores. Entre quienes estaban equipados en marzo 2004, un $51 \%$ de los primeros poseía un sistema anterior al año 2000 contra un $62 \%$ de los segundos.

Así mismo, un 46\% de becados declara resolver sus necesidades de acceso a internet gracias a los medios que provee la universidad, hecho que pone de relieve el rol compensatorio que la institución puede jugar en dicho campo. No obstante, cuando los estudiantes fueron invitados a pronunciarse sobre siete aspectos de la calidad de dicho acceso en sus respectivas facultades, se observaron diferencias marcadas y significativas entre unidades académicas. Globalmente, las facultades con altos aranceles ofrecen mejores condiciones según las apreciaciones estudiantiles.

El gráfico 1 nos muestra los resultados de un Análisis de Correspondencias Múltiples (ACM) realizado sobre algunas variables categoriales ${ }^{7}$. En términos generales, el ACM muestra la precariedad relativa de los estudiantes titulares de una beca PAE. En efecto todas las variables, excepto el género, presentan relaciones significativas con la condición socioeconómica (a Khi2 p<0.001). No obstante, el interés del gráfico 1 es mostrar una cierta estructura en las relaciones entre las diferentes modalidades analizadas.

7 Este tipo de análisis tiene la ventaja de describir, de manera sintética, grandes conjuntos de datos por la vía de proyectar sobre un plano las diferentes inercias (o distancias a la situación de independencia) asociadas al conjunto de las modalidades de variables. En general, mientras mayor distancia al centro de gravedad, mayor contribución a la inercia total de la nube de puntos o intersecciones. Mientras mayor cercanía a un eje, mayor contribución a la interpretación de éste, es decir, a la expresión de una dimensión latente en el conjunto de datos. La intersección de los ejes corresponde a la situación de independencia y se constituye en la referencia de las distancias geométricas entre modalidades. 
Gráfico 1. Proyección sobre un plan factorial (ACM) de las variables referidas a equipamiento informático, género, y capital cultural y escolar de los estudiantes. Variable suplementaria: condición socioeconómica (2004, n=719).

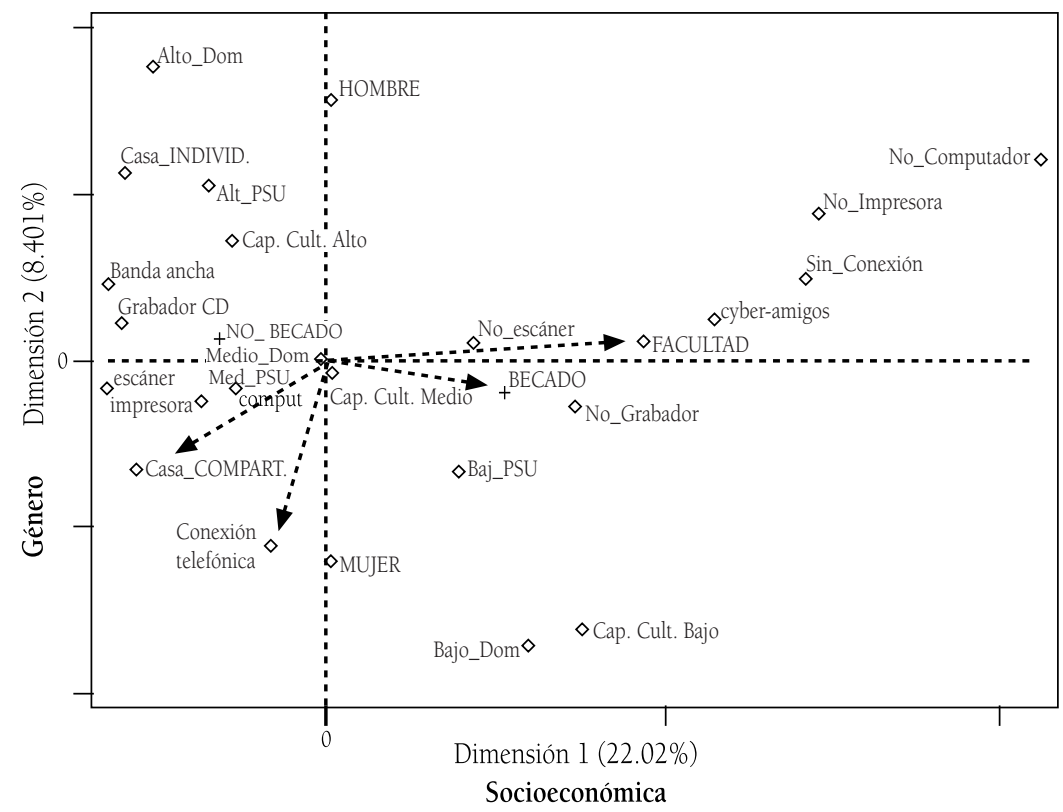

Para comprender mejor la relación entre los puntos en el plano se deben considerar los ángulos entre diferentes modalidades, en donde el vértice principal es el centro de gravedad del plano. Así, las modalidades que se encuentran cercanas a un ángulo de $90^{\circ}$ tienden a ser independientes unas de otras. Los ángulos agudos indican atracción y los obtusos repulsión.

En total, los dos ejes de representación o factores del ACM resumen un 30,4\% de la inercia total de la nube de puntos, lo que nos habla de por sí de una población de tendencia homogénea. La interpretación de los ejes escogidos permite hablar, por una parte, de una dimensión socioeconómica (eje horizontal) en donde a la izquierda del plano encontramos un buen nivel de capitales culturales materiales referidos a la informática (conexión en casa, banda ancha, equipamiento, por ejemplo), lo que, a su vez, se asocia con elevados capitales culturales familiares ( $>200$ libros en casa) y escolares (rango PAA-PSU >750 puntos). En el otro lado nos encontramos con las situaciones contrarias: menor equipamiento y, sobre todo, un acceso a internet restringido y dependiente (facultad y casa de amigos, 
parientes o cybercafés). Las modalidades intermedias (dominio medio, capital cultural medio, por ejemplo) aparecen próximas a la situación de independencia o perfil medio.

El segundo eje (vertical) aparece totalmente definido por la variable género, oponiendo nítidamente a hombres y mujeres. Es interesante notar cómo los niveles de dominio de internet declarados se asocian fuertemente a cada sexo para desventaja de las mujeres. Más interesante aún parece la cercanía entre la condición femenina y ciertas modalidades de acceso y conexión. En efecto, vemos cómo la modalidad "mujer" tiende a atraerse con variables como "conexión telefónica" y "acceso compartido con el resto de los miembros de la familia", modalidades que asignan una menor importancia a este tipo de capital.

La definición de la inercia de esta nube de puntos (modalidades) no consideró el hecho de que los estudiantes sean o no becados. Dicha variable aparece superpuesta en el plano (i.e. suplementaria) tendiendo a confirmar la visión ya descrita respecto del eje o dimensión socioeconómica.

\section{¿Cuán diferente es el equipamiento?}

Para profundizar en el análisis de las relaciones que el ACM tiende a poner de manifiesto, y así poder realizar inferencias válidas a partir de la muestra, se hicieron diferentes regresiones logísticas dicotómicas, lo que nos permitió testear relaciones estadísticamente significativas entre las variables, en condiciones comparables ${ }^{8}$. Globalmente es posible afirmar que:

8 Este tipo de regresión modeliza la probabilidad de ocurrencia de un hecho con relación a su contrario, bajo una ecuación del tipo: $E\left(y=11 \mathrm{x}_{1}\right)=\frac{\exp ^{\alpha+\Delta x_{1}}}{1+\exp ^{0+\Delta x_{1}}}$ donde $y$ es la variable a explicar (i.e. la probabilidad de que ella tome un cierto valor), $X$ es un vector de variables explicativas asociadas al individuo $i$; a y b representan coeficientes que se desea estimar, y donde $b$ es un vector de los mismos que expresa el impacto de las variables que componen $X$ sobre la probabilidad buscada. Para cada una de ellas se obtiene un coeficiente logit que permite estimar el Odds Ratio $\left(\frac{p(y=11 \mathrm{x}=1)}{1-p(y=11 \mathrm{x}=1)}\right) /\left(\frac{p(y=1 \mathrm{x}=0)}{1-p(y=11 \mathrm{x}=0}\right)$ de cada modalidad respecto de su referencia. Más detalles en tabla 4. 
i. Las variables que expresan el capital cultural material informático de los estudiantes se encuentran fuertemente ligadas entre ellas y, a su vez, a la condición socioeconómica, con excepción del hecho de poseer o no un computador. Esto último puede ser visto como algo positivo, pues puede pensarse que el acceso a dicha herramienta tiende a democratizarse.

ii. La posesión de impresora está fuertemente ligada a tener o no computador, pero, al igual que para poseer un escáner, ninguno de esos tres bienes aparece significativamente relacionado con la condición socioeconómica. Por el contrario, la posesión de un grabador de $\mathrm{CD}$ resulta fuertemente ligada a ésta, a favor de los no becados. Ello podría estar indicando los lazos y determinaciones potencialmente existentes entre prácticas y destrezas (capitales incorporados) y recursos (capitales materiales). El grabador de CD, en 2004 al menos, representaba un nivel superior de compenetración con los útiles informáticos y con el acopio, búsqueda y transmisión de datos.

iii. Se confirma una fuerte asociación entre becados y las variables condiciones deacceso (facultades)yno posesión de conexiónenel hogar.

iv. Tanto los niveles bajo e intermedio de capital cultural familiar, así como el rango inferior de puntaje PAA-PSU, continúan, en condiciones controladas, presentando relaciones más bien fuertes con la condición socioeconómica baja (a p<0.0001, $\mathrm{p}<0.03$ y $\mathrm{p}<0.15$, respectivamente).

v. Por último, el dominio declarado de internet, que podría ser visto como un indicador sintético de capital cultural incorporado para esa herramienta, pierde significación en su relación con la variable socioeconómica. En otras palabras la probabilidad de que los becados se declaren en un nivel determinado de dominio (en este caso "bajo"), deja de ser importante cuando se controlan simultáneamente otros factores. De manera similar, para la variable "actitud hacia internet" no se aprecian diferencias significativas. En definitiva, la relación que en términos brutos muestra el gráfico 1 entre la condición de becado y la modalidad "bajo dominio" dependería fundamentalmente del hecho de que este segmento de la población presenta un menor equipamiento y un menor capital 
cultural familiar. Por último, la condición de género se relaciona fuertemente con esta categoría en condiciones comparables.

\section{Lugares de adquisición de capitales}

Otro flanco de observación relevante son los espacios de socialización en los cuales los estudiantes declaran haberse familiarizado con la informática. Ante la pregunta "tus primeros contactos y aprendizajes en relación con la computación los hiciste en..." las respuestas obtenidas según categoría socioeconómica dejan entrever diferencias más o menos importantes respecto de los lugares y momentos donde ello ocurre. Mientras entre los no becados dos estudiantes sobre cinco declaran haber establecido sus primeros contactos con la informática en la casa familiar, entre los becados sólo uno de cuatro hace la misma afirmación. Así mismo, mientras para los primeros el 45\% aprende en el sistema educativo, y entre ellos el $71 \%$ lo hace durante la enseñanza básica, para los becados esas cifras corresponden al 56\% y 45\%, respectivamente. Tales observaciones se resumen en el gráfico 2 .

Las cifras de este gráfico son consistentes con las diferencias significativas entre ambas categorías respecto de la variable "años de experiencia en internet". En términos brutos los becados poseen en promedio de 4,2 años de experiencia declarada (desviación estándar - d.e.-2,05) mientras que los no becados les aventajan con 5,2 años en promedio (d.e. 2,02). Al mismo tiempo, no existe efecto de interacción verificable entre género y condición socioeconómica, y la variable género no presenta un impacto diferenciado sobre los años de experiencia. Cuando se controlan sexo, edad, capital escolar y capital cultural familiar, la diferencia por condición socioeconómica se reduce pero continúa siendo negativa y significativa $(\mathrm{p}<0.01)$.

El gráfico 2 reafirma también el valor del sistema educativo como espacio de adquisición de capitales culturales de todo orden, y como potencial palanca de disminución de las desigualdades y de nivelación entre individuos respecto de la brecha digital (Hopenhayn, 2003). Notemos, además, el rol que ejerce un cierto nivel de capital social para el 
caso de los estudiantes de menores recursos. Un 15\% de becados contra un 10\% de no becados declara haber tomado contacto con la informática gracias a parientes o amigos. El acceso a internet al momento de la encuesta indica una situación similar. Sin embargo, y al mismo tiempo, las mujeres, especialmente las becadas, se ausentan significativamente de esta modalidad capital social (casa de amigos o parientes) ${ }^{9}$.

Gráfico 2. Distribución de estudiantes respecto de los lugares en los cuales declaran haber realizado sus primeros aprendizajes respecto de la informática, según condición socioecoómica. (2004; n=719)
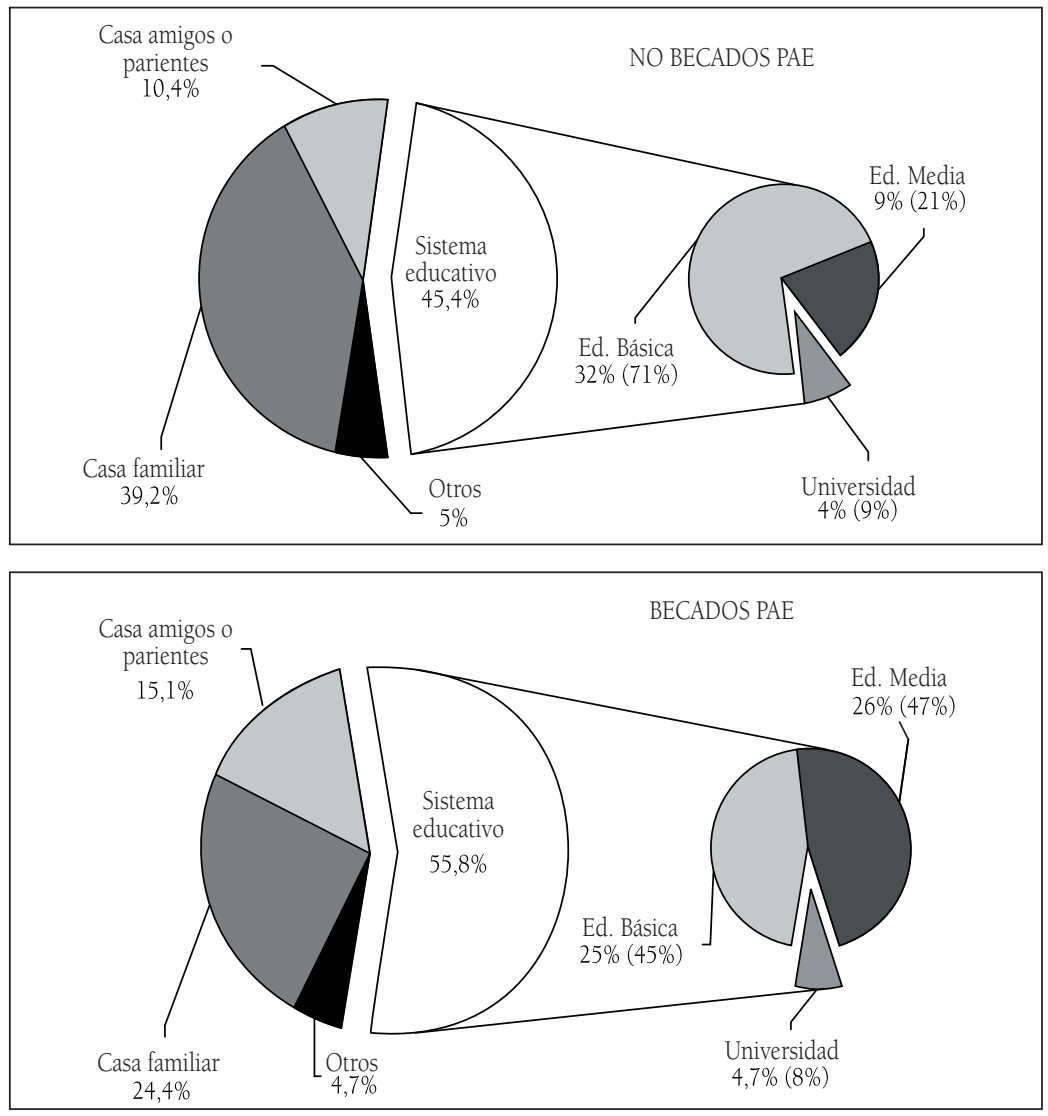

9 Ello es consistente con los resultados europeos del Programme for Indicators of Student Assessment (PISA) 2003, en donde los hombres (15 años) declaran aprender solos o con amigos, mientras que las mujeres lo hacen en la escuela o en la familia (EURIDYCE, 2005). 
Por último, el casi 5\% que declara haber tomado contacto en la universidad se distingue por ser mayor ( 25 años promedio) y evidencia la evolución de los niveles de penetración de la informática.

\section{¿Comportamientos diferentes?}

La constatación de diferencias entre becados y no becados en términos de equipamiento informático y de acceso y experiencia en internet nos impulsa a verificar la existencia de comportamientos diferentes en el uso de estos instrumentos. El gráfico 3 ilustra las distribuciones de tiempo declarado de conexión semanal a internet (todo tipo de usos) a partir de la intersección entre las variables género y condición socioeconómica.

En general, el total de los interrogados se conecta en promedio 8,9 horas semanales (d.e. 7,4). El rango interquartil -menos sensible a los valores extremos- es similar (siete horas). Ello nos habla de una importante diversidad en los tiempos de uso. Por otra parte, para ambos segmentos socioeconómicos de la muestra la variable género establece fuertes diferencias. Mientras los hombres no becados se conectan en promedio 11,3 horas por semana (d.e.10), los hombres becados lo hacen 8,6 (d.e. 7,0). Por su parte las mujeres se subdividen en 8,9 horas promedio y 6,3 horas, respectivamente, tendiendo a presentar conductas más homogéneas (d.e. de 6,2 y 4,5).

De manera evidente, resulta pertinente preguntarse por un eventual efecto de interacción en desmedro de las mujeres becadas, es decir, saber si su baja conexión se debe al hecho de ser mujeres, pero si se acentúa tanto más por poseer una condición socioeconómica desmejorada.

El gráfico 3 pone especialmente de relieve la fuerte diferencia (bruta) en tiempos de conexión entre becados y no becados: 7,3 horas contra 9,9 en promedio, respectivamente (d.e. de 5,8 y 8,1). Esta constatación nos devuelve a nuestra pregunta central: ¿se deben dichas diferencias a la primacía de capitales culturales incorporados, o bien 
son principalmente reflejo de las diferencias de capitales culturales materiales? ¿Se trata de problemas de disposición o de equipamiento, o de ambos?

Gráfico 3: Perfiles de las distribuciones de tiempo de conexión semanal a internet según condición socioeconómica y género (en horas) (2004; n= 719)

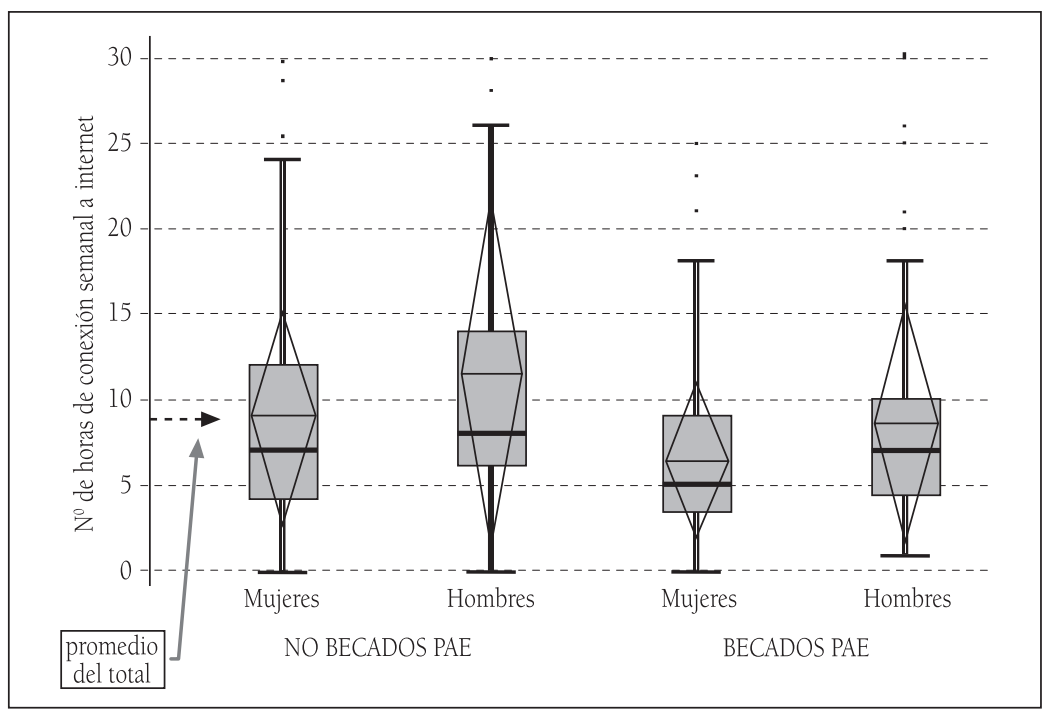

Generamos cuatro modelos explicativos para la variable "tiempo de conexión semanal" y consideramos tres subgrupos de variables explicativas y/o de control. Este análisis, mediante regresión múltiple ${ }^{10}$, permite -en términos ceteris paribus-responder de manera más precisa las preguntas que nos interesan. Sus resultados son expuestos en detalle en la tabla 1.

10 Bajo el método OLS (ordinary less squares) a través de una ecuación del tipo $y=a+b X_{i}+c Z_{i}+a T_{i}+v$, en donde $y$ corresponde al número de horas de conexión; $a, b, c$ $y d$ son vectores de coeficientes a estimar, $X, Z$ y $T$ son vectores que agrupan las características individuales, las actitudes y el equipamiento; $y$ donde $v$ corresponde a un término aleatorio de error. 
Tabla 1. Cuatro modelos de regresión lineal multivariable explicativos del tiempo de conexión semanal a internet declarado por los estudiantes. En horas a la semana (2004; $\mathrm{n}=719)$

\begin{tabular}{|c|c|c|c|c|c|c|c|c|c|}
\hline & \multicolumn{2}{|c|}{ Modelo $\mathrm{N}^{0} \mathrm{l}$} & \multicolumn{2}{|c|}{ Modelo $\mathrm{N}^{\circ} 2$} & \multicolumn{2}{|c|}{ Modelo $\mathrm{N}^{0} 3$} & \multicolumn{2}{|c|}{ Modelo $\mathrm{N}^{\circ} 4$} \\
\hline & & \multicolumn{2}{|c|}{\begin{tabular}{|l|} 
Características \\
individuales
\end{tabular}} & \multicolumn{2}{|c|}{\begin{tabular}{|l|} 
Características \\
individuales + \\
Actitudes hacia \\
internet
\end{tabular}} & \multicolumn{2}{|c|}{\begin{tabular}{|l|} 
Características \\
individuales + \\
Condiciones de \\
acceso
\end{tabular}} & \multicolumn{2}{|c|}{$\begin{array}{l}\text { Características } \\
\text { individuales + } \\
\text { Condiciones } \\
\text { de acceso + } \\
\text { Actitudes hacia } \\
\text { internet }\end{array}$} \\
\hline \multicolumn{2}{|c|}{$\mathrm{R}^{2}$ Ajustado (\% de varianza explicada) } & \multicolumn{2}{|l|}{0,08} & \multicolumn{2}{|c|}{0,12} & \multicolumn{2}{|l|}{0,23} & \multicolumn{2}{|c|}{0,25} \\
\hline & Constante & 9,1 & $(2,54)^{m+*}$ & 11,0 & $(2,64)^{s, n}$ & 7,9 & $(2,42)^{n+k}$ & 9,4 & $(2,53)^{* \cdots}$ \\
\hline $\begin{array}{l}\text { MODALIDAD DE } \\
\text { REFERENCIA }\end{array}$ & $\begin{array}{l}\text { MODALIDAD(es) } \\
\text { ACTIVA(s) }\end{array}$ & & & & & & & & \\
\hline Mujer & Hombre & 2,0 & $(0,54)^{* * *}$ & 1,3 & $(0,54)^{* n}$ & 2,0 & $(0,50)^{*+*}$ & 1,4 & $(0,51)^{*+*}$ \\
\hline No becado PAE & Becado PAE & $-1,7$ & $(0,58)^{* *}$ & $-1,6$ & $(0,57)^{* n+\infty}$ & 0,6 & $(0,58)$ & 0,6 & $(0,57)$ \\
\hline- & Edad al 01/03/2004 & $-0,1$ & $(0,12)$ & $-0,1$ & $(0,11)$ & 0,0 & $(0,11)$ & 0,0 & $(0,11)$ \\
\hline \multirow{2}{*}{$\begin{array}{l}\text { Nivel en la carrera: } 1^{\text {er }} \\
\text { Año U. de Chile }\end{array}$} & $2^{\circ}$ Año U & $-0,2$ & $(0,72)$ & 0,0 & $(0,71)$ & $-0,2$ & $(0,67)$ & 0,1 & $(0,66)$ \\
\hline & $3^{\text {er Año U o más }}$ & 0,9 & $(0,82)$ & 0,9 & $(0,80)$ & 0,7 & $(0,75)$ & 0,7 & $(0,74)$ \\
\hline \multirow{2}{*}{$\begin{array}{l}\text { Rango del ptje. promedio } \\
\text { PAA-PSU (Leng \& Mat) } \\
\text { Medio }\end{array}$} & Puntaje BAJO (<680 pts.) & $-0,02$ & $(0,65)$ & $-0,3$ & $(0,63)$ & 0,7 & $(0,60)$ & 0,4 & $(0,59)$ \\
\hline & Puntaje ALTO (>750 pts.) & 1,1 & $\begin{array}{l}(0,71) \\
(<)\end{array}$ & & $(0,70)$ & 0,7 & $(0,66)$ & 0,6 & $(0,65)$ \\
\hline- & $\begin{array}{l}\text { Años de expriencia en uso } \\
\text { de internet }\end{array}$ & 0,5 & $(0,14)^{* *}$ & & $(0,15)$ & 0,3 & $(0,13)^{* *}$ & 0,0 & $(0,14)$ \\
\hline \multirow{2}{*}{$\begin{array}{l}\text { Libros en casa familiar } \\
(>200)\end{array}$} & \begin{tabular}{|l|}
$<50$ libros \\
\end{tabular} & $-2,0$ & $(0,88)^{* *}$ & $-1,7$ & $(0,86)^{38}$ & $-1,0$ & $(0,81)$ & $-0,8$ & $(0,80)$ \\
\hline & Entre 50 y 200 libros & $-0,3$ & $(0,59)$ & $-0,3$ & $(0,58)$ & $-0,0$ & $(0,54)$ & 0,0 & $(0,54)$ \\
\hline \multirow{3}{*}{$\begin{array}{l}\text { Acceso prioritario en: } \\
\text { casa (pieza individual) }\end{array}$} & En casa amigos/cyber/etc. & & & & & $-4,8$ & $(1,20)^{* * *}$ & $-4,2$ & $(1,19)^{\prime \prime \prime}$ \\
\hline & \begin{tabular}{|l|} 
Facultad \\
\end{tabular} & & & & & $-4,6$ & $(0,93)^{k+k}$ & $-4,3$ & $(0,92)^{* *}$ \\
\hline & Casa (espacio compartido) & & & & & $-3,8$ & $(0,68)^{*+*}$ & $-3,6$ & $(0,67)^{* *}$ \\
\hline \multirow{2}{*}{$\begin{array}{l}\text { Conexión telefónica en } \\
\text { la casa }\end{array}$} & Sin conexión & & & & & $-0,6$ & $(0,89)$ & $-0,5$ & $(0,88)$ \\
\hline & Conexión banda ancha & & & & & 4,3 & $(0,64)^{* * * *}$ & 4,3 & $(0,63)^{* *}$ \\
\hline \multirow[t]{2}{*}{ Actitud entusiasta } & Rechazo & & & $-2,0$ & $\begin{array}{l}(1,36) \\
(<)\end{array}$ & & & $-0,9$ & $(1,26)$ \\
\hline & Indiferencia / Aceptación & & & $-0,5$ & $(0,73)$ & & & $-0,5$ & $(0,68)$ \\
\hline \multirow{2}{*}{$\begin{array}{l}\text { Dominio declarado de } \\
\text { internet (MEDIO) }\end{array}$} & BAJO dominio & & & & $(0,73)^{n+\infty}$ & & & $-2,3$ & $(0,68)^{\prime \prime \prime}$ \\
\hline & Dominio ELEVADO & & & & $(0,74)^{n+s+}$ & & & 2,4 & $(0,69)^{\prime \prime \prime}$ \\
\hline
\end{tabular}

Cada modelo o columna considera: a) el coeficiente respectivo para cada variable y/o modalidad; b) el error tipo de dicho coeficiente (entre paréntesis); y c) su significación estadística expresada en términos de probabilidad de error $\rightarrow$ Ningún símbolo $=$ no significativo; $("<)=p<0,15^{* *}=p<0,05 ;{ }^{* * *}=p<0,01$ o menos.

En el Modelo No 2 los estudiantes que poseen una beca del Programa de Atención Socioeconómica (PAE) se conectan, en promedio, 1,6 menos que los estudiantes no becados, ello a condiciones comparables con respecto a todos los otros factores o variables explicativas incluidas en ese modelo. Dicha situación es altamente significativa desde un punto de vista estadístico $(\mathrm{p}<0,01)$. Por su parte, la varianza explicativa por el modelo $\mathrm{N}^{\circ} 2$ equivale a un $12,3 \%\left(\mathrm{R}^{2}\right.$ aj. $\left.=0,123\right)$ de las diferencias de tiempo semanal de conexión a internet constatadas entre los 719 individuos de la muestra. En ninguno de los modelos se aprecian fenómenos de colinealidad importantes $(\mathrm{IFV}<2,95)$. 
Numerosas informaciones pueden ser extraídas de este análisis, sin embargo, retendremos la evolución de varianza explicada $\left(R^{2}\right)$ y, sobre todo, la modificación de impactos que se produce en la medida en que diferentes variables son consideradas. En efecto, mientras los modelos 1 y 2 arrojan una diferencia significativa de horas de conexión entre becados y no becados (en promedio 1,7 y 1,6 horas menos para los primeros comparados con los segundos), cuando se controlan las condiciones de acceso y de conexión (modelos 3 y 4) dicha diferencia disminuye, cambia de sentido y, sobre todo, pierde significación estadística, indicándonos que es pertinente retener la hipótesis nula de inexistencia de diferencias significativas entre ambas categorías, en condiciones controladas. Al mismo tiempo, la ganancia en términos de explicación de la varianza aumenta al doble, reforzando la idea de que son las condiciones de acceso las que determinan los tiempos de uso.

Una situación similar ocurre (modelos 1 y 2) con la categoría inferior de capital cultural familiar, la que, no obstante, muestra un impacto no despreciable de menos dos horas en promedio a similar condición socioeconómica. Este efecto es absorbido luego por las condiciones de acceso y conexión, lo que indica que es necesario considerar especialmente a la población de menores capitales culturales familiares, pues ella presenta, al mismo tiempo, más probabilidades de poseer menos capitales materiales.

Estos resultados nos indican que los estudiantes de condición socioeconómica desmejorada se conectan menos que los otros, no por una disposición particular a ello, sino que porque en promedio poseen menos capitales materiales. Por cierto, esto no exime la existencia de un cierto nivel de brecha digital, más bien ayuda a resituarla. Es indispensable recordar que nuestra población de origen es de por sí una muestra sesgada de la sociedad chilena y que además puede ser más homogénea que la de otras instituciones.

Dicho de otro modo, las constataciones de la tabla 1 podrían verse relativizadas si se considera una población estudiantil más representativa del total nacional o bien de otro tipo de institución. Desde un punto de 
vista de política universitaria, estos resultados indicarían que un énfasis fundamental se relaciona con los capitales culturales materiales que las instituciones ponen al servicio de sus estudiantes en aras de lograr equidad durante la experiencia universitaria y frente a las exigencias académicas a los que éstos se ven enfrentados.

Por otro lado, gracias a las variables que reflejan las actitudes y percepciones respecto de internet, vemos como los años de experiencia pierden impacto cuando se controla el dominio declarado. Por cierto, ambas variables se encuentran correlacionadas entre sí, sin embargo, adquirir un cierto dominio y/o una cierta confianza puede jugar un rol compensatorio frente a los años de uso, lo que puede lograrse mediante cursos, nivelaciones, entre otros.

Por último, en condiciones comparables (modelo 4), las mujeres, continúan conectándose significativamente menos que los hombres $(-1,4$ horas en promedio). Si consideramos, en sentido inverso, el sesgo natural de nuestra población, resulta posible pensar que esta brecha tiende a ser aún más marcada en la sociedad en general.

\section{Los tipos de uso dados a internet}

La hipótesis según la cual la intensidad de uso de internet en tiempo sería diferente a partir de la condición socioeconómica de los estudiantes no aparece validada. Resulta entonces necesario interrogarse sobre otros parámetros de la calidad de dicho uso.

Para ello se analizaron las respuestas de los estudiantes ante variadas proposiciones de uso de internet y frente a las cuales éstos se pronunciaron sobre la frecuencia de dicha práctica en forma de escala de Likert en cinco niveles. A partir de dicha información se crearon también reagrupamientos utilizando como indicador de pertinencia y fiabilidad el coeficiente Alpha de Cronbach (>0.5).

Bajo esa lógica se creó la variable "uso total de internet", correspondiente al promedio simple del conjunto de valores dados 
por cada individuo a todas las opciones de respuesta. Ella puede ser pensada como un indicador de la utilización más o menos integral de internet. Es posible postular que, mientras más elevado sea su valor, los estudiantes estarán obteniendo un mayor provecho de ésta y mayor será su disposición y capacidad a servirse integralmente de la misma.

La tabla 2 presenta los parámetros de ubicación y dispersión para las distintas variables, respecto de la frecuencia de uso por actividad o grupo de actividades.

Tabla 2. (1) Media, límites de confianza de la media $(p>0,05)$ y desviación estándar para las diferentes variables de uso de internet. Escala de actitudes de 1 (No lo hago nunca cuando estoy conectado) a 5 (... casi todas o todas las veces cuando estoy conectado). (2) Matriz de correlaciones de Rango de Spearman. Significativas en cursiva. (2004; n= 719)

\begin{tabular}{|c|c|c|c|c|c|c|c|c|c|c|c|}
\hline & $\begin{array}{l}\text { Comuni- } \\
\text { cación }\end{array}$ & ESTUDIAR & $\begin{array}{l}\text { Música } \\
\text { y bajar } \\
\text { archivos }\end{array}$ & $\begin{array}{c}\text { Temas } \\
\text { descubrir }\end{array}$ & $\begin{array}{c}\text { Infos } \\
\text { noticias }\end{array}$ & E-mail & Contactos & $\begin{array}{l}\text { Buscar } \\
\text { trabajo }\end{array}$ & Comprar & Trámites & $\begin{array}{l}\text { Uso } \\
\text { total }\end{array}$ \\
\hline Media & 2,2 & 4,0 & 3,4 & 3,3 & 3,1 & 4,6 & 2,9 & 1,6 & 1,9 & 3,0 & 2,9 \\
\hline (LC 95\%) & $(0,06)$ & $(0,06)$ & $(0,08)$ & $(0,06)$ & $(0,07)$ & $(0,06)$ & $(0,08)$ & $(0,07)$ & $(0,07)$ & $(0,07)$ & $(0,04)$ \\
\hline d.e. & 0,8 & 0,8 & 1,1 & 0,8 & 0,9 & 0,8 & 1,2 & 0,9 & 1,0 & 0,9 & 0,5 \\
\hline $\begin{array}{l}\text { Comuni- } \\
\text { cación }\end{array}$ & 1 & & & & & & & & & & \\
\hline ESTUDIAR & $-0,03$ & 1 & & & & & & & & & \\
\hline $\begin{array}{l}\text { Bajar } \\
\text { música }\end{array}$ & 0,45 & $-0,04$ & 1 & & & & & & & & \\
\hline $\begin{array}{l}\text { Temas } \\
\text { descub. }\end{array}$ & 0,13 & 0,16 & 0,27 & 1 & & & & & & & \\
\hline $\begin{array}{l}\text { Leer info. y } \\
\text { noticias }\end{array}$ & 0,30 & 0,03 & 0,38 & 0,41 & 1 & & & & & & \\
\hline E-mail & 0,24 & 0,07 & 0,10 & 0,07 & 0,22 & 1 & & & & & \\
\hline Contactos & 0,15 & 0,17 & 0,07 & 0,24 & 0,21 & 0,08 & 1 & & & & \\
\hline $\begin{array}{l}\text { Buscar } \\
\text { trabajo }\end{array}$ & 0,10 & 0,02 & 0,06 & 0,18 & 0,26 & 0,07 & 0,14 & 1 & & & \\
\hline Comprar & 0,24 & 0,02 & 0,31 & 0,22 & 0,38 & 0,14 & 0,06 & 0,22 & 1 & & \\
\hline Trámites & 0,05 & 0,22 & 0,03 & 0,24 & 0,24 & 0,13 & 0,18 & 0,18 & 0,19 & 1 & \\
\hline Uso total & 0,70 & 0,14 & 0,66 & 0,53 & 0,69 & 0,32 & 0,37 & 0,33 & 0,52 & 0,31 & 1 \\
\hline
\end{tabular}

Entre los valores 1 y 5 , y en promedio para la muestra, las actividades ligadas a utilizar internet para fines temático-exploratorios (Temas-descubrir) se ubican en un valor de 3,3. El límite de confianza (función de la dispersión y del tamaño de la muestra) corresponde al valor que, sumando y restando al promedio, indica la zona de confianza de dicho valor ( entre 3,3 $\pm 0,06$ ) lo que permite comparar su significación estadística con las restantes variables. Entre "Bajar música" y "Leer informaciones y noticias" existe una variación conjunta significativa de 0,38 e indica que quienes realizan una de esas actividades tienden también a realizar la otra. 
Como se aprecia, las actividades más frecuentes que los estudiantes de la muestra declaran realizar en internet dicen relación con el estudio (todo tipo de cursos) y con el envío y la lectura de emails. Ello es globalmente concordante con otros estudios nacionales e internacionales (Jaramillo et al., 2002; Godoy y Herrera, 2004). Por su parte, la búsqueda de trabajo y la de informaciones para realizar compras figuran entre las menos frecuentes.

El gráfico 4 muestra estas mismas variables sometidas a un análisis canónico discriminante (ACD). Se trata de testear diferencias en los tipos de uso de internet a partir de las categorías sociales más relevantes.

Gráfico 4. Plan factorial de ejes canónicos representando los diferentes usos dados a internet a partir de un $\mathrm{ACD}$, usando como variable de clasificación la interacción entre las variables género y la condición socioeconómica. (2004; n= 719)

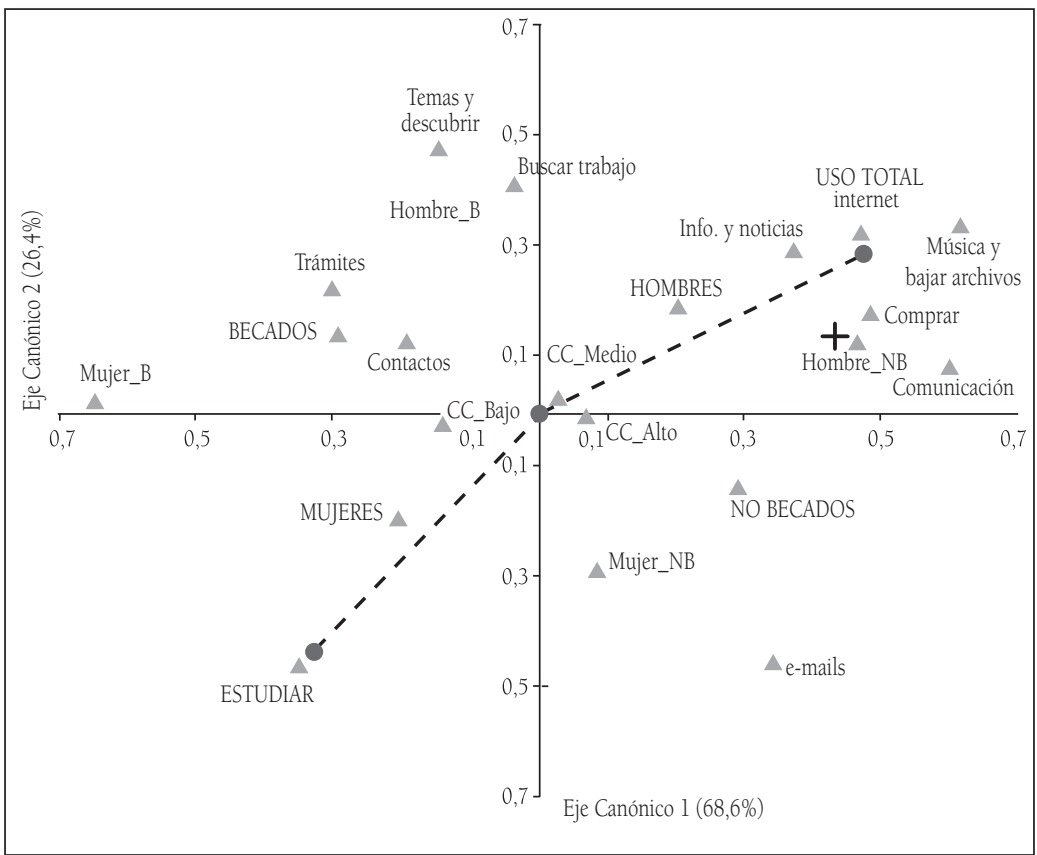


El interés de un análisis discriminante proviene del hecho de distinguir, entre un conjunto de variables -en este caso las frecuencias declaradas frente a las proposiciones realizadas-, cuáles sirven para diferenciar lo más posible los diferentes grupos que se desea caracterizar. Ello nos permite saber si entre hombres y mujeres, de condición socioeconómica desmejorada o no, existen tipos de uso de internet diferentes. Se trata de confirmar o refutar la existencia de tendencias y/o de comportamientos diferenciados, incluso si el valor explicativo total del análisis es reducido, como puede ocurrir en poblaciones más bien homogéneas como es el caso ${ }^{11}$. Por otra parte, la ventaja del ACD es que nos permite visualizar gráficamente el conjunto de variables utilizadas, proyectar otras sobre el plano y, a la vez, comprender mejor las estructuras internas no aparentes que las relacionan; la sola matriz de correlaciones (tabla 2) no nos permite ver dichas estructuras.

El plano creado por los dos ejes canónicos de este análisis muestra las atracciones, independencias y oposiciones entre variables. En cuanto a los usos, mientras la variable "estudiar" se ubica claramente en el cuadrante inferior izquierdo, la variable síntesis "uso total" se ubica en el cuadrante opuesto. Entre ambas frecuencias de uso se dibuja un ángulo de casi $180^{\circ}$ y sobre ese mismo eje tiende a ubicarse la variable "género". Al mismo tiempo, usos como "grabar música y bajar archivos" o "búsqueda de informaciones y noticias" aparecen bien correlacionados con "uso total". Considerando que el intercambio de información y de bienes (culturales) es propio de internet, notamos la fuerte correlación existente entre ellas y "uso total" (tabla 2). Una proyección adicional (símbolo cruz) indica que el mayor número de horas de uso tiende también a colocarse en ese mismo cuadrante.

Globalmente, se aprecia un cruce diagonal a través de una suerte de gran $\mathrm{X}$ inclinada en el plano, la que opone por una parte a hombres y mujeres, y, por otra, a becados y no becados. Así, por ejemplo, vemos que la prioridad que las mujeres declaran asignar al

11 La distancia de Mahalanobis es significativa $(\mathrm{p}<0.0001)$ para las cuatro categorías. Promedio $\mathrm{R}^{2}=0.03$, significativo para todos los tests. Los análisis de varianza por tipo de uso son significativos a $\mathrm{p}<0.05$, salvo para "trabajo" $(\mathrm{p}<0.08)$. 
uso de internet como herramienta para sus estudios parece no dejar lugar a dudas. Por su parte, los hombres no becados se distinguen bien por un uso integrado e integral de internet. Integrado, porque las actividades de comunicación recreativa y otras, como el acceso a ciertos bienes (información, música, buscar ofertas, entre otras) estarían bien incorporadas en sus vidas. Integral, porque un uso total más elevado supone una tendencia a practicar una gama más amplia de actividades en la red.

Una lectura diferente y no contradictoria con lo anterior visualiza lo que parece como una división entre aquellos para los cuales internet es una herramienta integrada de lleno en sus vidas y quienes tienen una relación menos intensa o más ocasional pero, a la vez, fuertemente funcional con ella. Los becados tenderían más bien a ubicarse en dichos cuadrantes (izquierda del plano), con la salvedad de un uso más intenso por parte de los hombres.

Finalmente, la tabla 3 presenta los resultados de diferentes regresiones, otorgando una vista global a partir de una gran cantidad de variables explicativas posibles. Ello permite comprender qué factores ejercen una influencia (positiva o negativa) en dichos usos, para, desde allí, extraer algunas conclusiones respecto de nuestras hipótesis.

Tabla 3. Modelos de regresión lineal que incluyen las variables con un impacto y/o relación significativos sobre las frecuencias declaradas de uso de internet para cuatro tipos de actividades (escala 1 a 5; selección stepwise) (2004; n = 719).

\begin{tabular}{|c|c|c|c|c|c|c|c|}
\hline \multicolumn{2}{|c|}{ Estudiar } & \multicolumn{2}{|c|}{ Comunicación } & \multicolumn{2}{|c|}{ Música y bajar archivos } & \multicolumn{2}{|c|}{ Uso total } \\
\hline \multicolumn{2}{|c|}{$\mathrm{R}^{2}$ aj. (\%) $4,8 \%$} & \multicolumn{2}{|c|}{$\mathrm{R}^{2}$ aj. (\%) $16,9 \%$} & \multicolumn{2}{|c|}{$\mathrm{R}^{2}$ aj. (\%) $22,04 \%$} & \multicolumn{2}{|c|}{$\mathrm{R}^{2}$ aj. (\%) $19,01 \%$} \\
\hline Constante & $+3,3 * * *$ & $\longrightarrow$ & $+2,6 * * *$ & 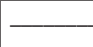 & $+3,6 * * *$ & $\longrightarrow$ & $+2,2 * * *$ \\
\hline Hombre & $-0,3 * * *$ & $\begin{array}{l}\text { Acceso: } \\
\text { Individual en } \\
\text { casa }\end{array}$ & $+0,3^{* * *}$ & $\begin{array}{l}\text { Conexión } \\
\text { ancha }\end{array}$ & $a+0,5 * * *$ & Hombre becado & $+0,2 * * *$ \\
\hline $\begin{array}{l}\text { Conexión } \\
\text { telefónica }\end{array}$ & $+0,2 * * *$ & $\begin{array}{l}\text { Actitud } \\
\text { aceptación de } \\
\text { internet }\end{array}$ & $+0,2 * *$ & Acceso: fa & $-0,5 * * *$ & $\begin{array}{l}\text { Conexión banda } \\
\text { ancha }\end{array}$ & $+0,2 * * *$ \\
\hline $\begin{array}{l}\text { Actitud } \\
\text { aceptación }\end{array}$ & $+0,2 *$ & $\begin{array}{l}\text { Conexión ban } \\
\text { ancha }\end{array}$ & $a+0,2 * * *$ & Hombre & $+0,4 * * *$ & $\begin{array}{l}\text { Acceso: } \\
\text { Individual en } \\
\text { casa }\end{array}$ & $+0,2 * * *$ \\
\hline
\end{tabular}




\begin{tabular}{|c|c|c|c|c|c|c|c|}
\hline Becado & $+0,1 *$ & Hombre & $+0,1 * *$ & $\begin{array}{l}\text { Acceso: cyber- } \\
\text { amigos-etc. }\end{array}$ & $-0,3 * *$ & $\begin{array}{l}\text { Actitud } \\
\text { aceptación de } \\
\text { internet }\end{array}$ & $+0,1 * *$ \\
\hline $\begin{array}{l}\text { Años de exp. } \\
\text { internet }\end{array}$ & $+0,03 * *$ & Acceso: faculta & $d-0,1 * *$ & Hombre becado & $+0,2 * *$ & $\begin{array}{l}\text { Hombre no } \\
\text { becado }\end{array}$ & $+0,1 * * *$ \\
\hline \multirow[t]{5}{*}{ Edad } & $+0,02 *$ & Mujer becada & $-0,1 *$ & $\begin{array}{l}\text { Alto puntaje } \\
\text { PSU-PAA }\end{array}$ & $-0,1>^{*}$ & \begin{tabular}{|l|} 
Alto puntaje \\
PSU-PAA
\end{tabular} & $-0,1^{* * *}$ \\
\hline & & $\begin{array}{l}\text { Años de exp. } \\
\text { internet }\end{array}$ & $+0,1 * * *$ & $\begin{array}{l}\text { Actitud } \\
\text { aceptación de } \\
\text { internet }\end{array}$ & $+0,1 *$ & $\begin{array}{l}\text { Capital cultural } \\
\text { BAJO }\end{array}$ & $-0,1 *$ \\
\hline & & Edad & $-0,05 * * *$ & $\begin{array}{l}\text { Capital cultural } \\
\text { MEDIO }\end{array}$ & $+0,2 * *$ & $\begin{array}{l}\text { Años de exp. } \\
\text { internet }\end{array}$ & $+0,1 * * *$ \\
\hline & & & & $\begin{array}{l}\text { Años de exp. } \\
\text { internet }\end{array}$ & $+0,1 * * *$ & & \\
\hline & & & & Edad & $-0,1 * * *$ & & \\
\hline
\end{tabular}

$>^{*}: \mathrm{p}<0,15 ; *: \mathrm{p}<0,1 ; * *: \mathrm{p}<0,05 ; *^{* *}: \mathrm{p}<0,01$ o menos. Para cada modelo, las variables deben ser consideradas en conjunto y cada una en relación con las opciones ausentes o modalidades de referencia, salvo que se trate de variables continuas (experiencia en internet o edad). Por ej.: Banda ancha se compara a la suma de quienes no poseen conexión y de quienes poseen conexión telefónica. Nota: la variable "comunicación" no incluye "e-mail" y en este caso "uso total" se calculó sin la variable "estudiar".

Hemos utilizado como opción de análisis la selección "paso a paso" (stepwise), puesto que nuestro principal interés es comprender las relaciones más relevantes hacia la conducta indicada. La tabla 3 muestra sólo las variables retenidas para cada modelo.

Correlación no significa causalidad. La atribución de ésta corresponde a las hipótesis, datos, evidencias o análisis previos de los investigadores. Por ejemplo, la "actitud de aceptación de internet" (variable muda, fusión de entusiasmo-aceptación y de indiferenciarechazo) no podría ser calificada como una variable causal de la frecuencia de usos. Resulta mucho más coherente postular que la relación ceteris paribus, cuya existencia el modelo pone en evidencia, indica una suerte de círculo virtuoso entre uso y valoración. Se valora la herramienta porque permite tales usos y viceversa. Por el contrario en el caso de la variable "banda ancha" y usos tales como "comunicación" o "bajar archivos..." existe a todas luces una relación causal en tanto la primera posibilita las segundas, lo que es coherente con otros estudios (Godoy y Herrera, 2004). 
Nuestra variable dependiente no es sino, en cada caso, una escala de frecuencias ordinal (individual o agregada) ${ }^{12}$. Por eso, lo importante es verificar un cierto orden de tamaño de impactos en los coeficientes anotados en cada modelo y, sobre todo, la importancia estadística de la relación y si ésta aumenta o disminuye el uso en cuestión $^{13}$. Cuando una variable no es retenida por el modelo ello indica ausencia de impacto, es decir, que la frecuencia de uso no es significativamente distinta entre sus modalidades o valores.

Las diferencias del gráfico 4 se ven confirmadas, esta vez en condiciones controladas. Para el caso de la variable "estudiar", la categoría "becado" y "conexión telefónica" indican una tendencia al uso de internet como útil de estudio, lo que resulta coherente con lo que puede suponerse de los comportamientos de los estudiantes ante la necesidad de priorizar según los medios de los cuales disponen. No es de extrañar que puedan existir estrategias de compensación por parte de esos estudiantes, en función de multiplicar las posibilidades de logro en lo académico y a sabiendas de que sus capitales sociales, escolares y culturales materiales pueden ser menores que el de sus pares con mejor estatus socioeconómico. La relación positiva entre los años de experiencia de internet y "estudiar" podría reforzar la idea de que la inexperiencia o la inseguridad limitan la eficacia del uso en este caso. Se usa más internet como herramienta de estudio porque se la domina mejor, lo que nos lleva a pensar que este uso "para los estudios" no debiera ser visto como algo banal o evidente, como no lo es tampoco poder usar eficazmente una biblioteca.

Respecto de nuestra problemática principal, sólo el modelo para la variable "estudiar" retiene la condición socioeconómica en su conjunto $(\mathrm{a} \mathrm{p}<0.1)$. Para los restantes tres modelos las diferencias aparecen en la interacción de ésta con el género y siempre para

12 En efecto, los porcentajes de varianza explicada aumentan para las variables agregadas (comparadas con "estudiar"), pues la variación total tiende a aumentar por construcción.

13 Las pruebas realizados para identificar posibles problemas de colinealidad entre variables indican que dicho fenómeno existe en rango medio para el modelo 3 entre las variables "aceptación de internet" y "años de experiencia" (ambas varían conjuntamente). Estos fenómenos no son lo suficientemente fuertes como para descartar los análisis. 
desventaja de las mujeres becadas. En general, los hombres becados se comportan de manera similar a los no becados, incluso declarando usos más intensos en algunos casos. Ello parece ser consistente con su media hora de conexión (gráfico 3), y estaría indicando que las diferencias de género tienden a acentuarse por el efecto de la condición socioeconómica, al menos en lo que a calidad (como multiplicidad) de uso se refiere. Se acumulan desventajas relativas en las mujeres becadas, puesto que ellas tienden a estar asociadas a otras categorías, tales como "bajo capital cultural", lo que, a su vez, las distancia de un uso más integral (modelo "uso total").

La variable "comunicación" presenta un comportamiento similar a "música y bajar archivos", en el sentido de que similares factores parecen influenciar a ambas. Es el caso de la experiencia en internet, donde la antigüedad como usuario aumenta la frecuencia declarada de tales usos (y, en general, el de todos los usos). Lo mismo ocurre con la variable "lugar prioritario de acceso", confirmando la idea de que para dedicarse a participar en foros y juegos en red, e invertir tiempo en comunicarse con otros, el equipamiento personalizado es fundamental.

Por su parte, el capital cultural general parece no ejercer influencia significativa alguna en la priorización de uso de internet para estudiar y tampoco influye en la variable "comunicación". Si lo hace con la variable "música..." en su modalidad "capital cultural medio", aumentando la frecuencia de uso en desmedro de quienes poseen un alto o un bajo capital cultural. Este resultado podría dar paso a ciertas hipótesis sobre el impacto de internet en los sectores medios y la percepción y uso que de ella existe. En ese mismo sentido, para el caso de estudiantes con alto capital escolar se evidencia una menor frecuencia significativa en el "uso total" y en "música..." al comparárseles con los estudiantes de puntajes medios y bajos en condiciones controladas. Ello parece indicar que un uso más intensivo declarado (autopercibido) de internet no se asocia con mayores capitales escolares ni culturales, lo que no quiere decir que no existan calidades y beneficios de uso diferente. 
Se confirma que los hombres se distancian de la opción "estudiar" al compararlos con las mujeres. Asimismo, el hecho de poseer una conexión de banda ancha en la casa no acerca los usuarios a dicha opción, lo que podría explicarse por la comodidad de acceso que permite extender los horizontes de utilización de internet y, al mismo tiempo, mantener la disponibilidad cada vez que ello sea necesario (para el estudio). El problema que plantea la "disponibilidad de la herramienta" no deja de tener repercusiones en lo que a disminución de brecha digital se refiere, pues es posible pensar que la situación tiende a ser más marcada aún en otros sectores. Una vez más, tiende a aparecer una relación de agregación, cuando no multiplicativa, entre capital cultural material e incorporado.

\section{Otras diferencias de uso y dominio}

Nuestra última área de análisis dice relación con el dominio y uso de ciertos programas informáticos, a partir de las respuestas a la pregunta: ¿con cuáles de los siguientes programas computacionales has hecho y terminado algún documento, archivo, producto o trabajo? (Word, Excel, Power Point, Otros (anótalos)). Los resultados obtenidos son detallados en las tablas 4 y 5 .

Tabla 4: Modelos resultantes de diferentes regresiones logísticas (selección stepwise) para explicar la probabilidad de "saber usar" los siguientes programas computacionales. Sólo se presentan los Odds Ratio de variables significativas. (2004; n=719)

\begin{tabular}{|c|c|c|c|c|c|c|c|c|c|}
\hline \multirow[t]{2}{*}{$\begin{array}{l}\text { Variable (mod. } \\
\text { referencia) }\end{array}$} & \multirow[t]{2}{*}{$\begin{array}{c}\text { Modalidades } \\
\text { activas }\end{array}$} & \multicolumn{2}{|r|}{ Word } & \multicolumn{2}{|r|}{ Excel } & \multicolumn{2}{|c|}{ Power Point } & \multicolumn{2}{|c|}{ Otro } \\
\hline & & (1) & (3) & (1) $(2$ & (3) & (1) (2) & (3) & (1) (2) & (3) \\
\hline \multirow{2}{*}{$\begin{array}{l}\text { Puntaje PSU - PAA } \\
\text { Ref. = MEDIO }\end{array}$} & \multirow{2}{*}{$\begin{array}{l}\text { Bajo < } 680 \text { pts. } \\
\text { Alto > } 750 \text { pts. }\end{array}$} & - & $0,4 \quad p<0,05$ & -0 & $0,8 \quad \mathrm{p}<0,12$ & $-0,5$ & $p<0,00$ & $\begin{array}{l}-\quad 0,5 \\
\end{array}$ & $\mathrm{p}<0,00$ \\
\hline & & + & 1,7 & + & $1,9 \quad \mathrm{p}<0,01$ & 1,0 & & $+\quad 1,4$ & $\mathrm{p}<0,11$ \\
\hline \multirow{6}{*}{$\begin{array}{l}\text { Área del } \\
\text { conocimiento de la } \\
\text { carrera } \\
\text { Ref-= tecnología }\end{array}$} & \multirow{6}{*}{$\begin{array}{l}\text { Agropecuaria, Artes } \\
\text { \& Arquitectura, } \\
\text { Cs. Naturales, } \\
\text { Humanidades, } \\
\text { Salud, Cs. Sociales }\end{array}$} & \multirow{6}{*}{\multicolumn{2}{|c|}{$\operatorname{nr}(*)$}} & & 2,0 & $\begin{array}{l}+2,8 \\
\end{array}$ & $\mathrm{p}<0,05$ & $\begin{array}{ll}-\quad 0,5 \\
\end{array}$ & \\
\hline & & & & $-\quad 0$ & $0,3 \quad \mathrm{p}<0,01$ & $\begin{array}{l}+\quad 0,9 \\
\end{array}$ & & $+\quad 1,8$ & \\
\hline & & & & +1. & 1,6 & - $\quad 0,7$ & & - $\quad 0,6$ & \\
\hline & & & & -0 & $0,2 \quad p<0,00$ & - 0,4 & $p<0,05$ & - $\quad 0,3$ & $\mathrm{p}<0,01$ \\
\hline & & & & & $0,6 \quad p<0,13$ & $+1,7$ & & 0,1 & $\mathrm{p}<0,00$ \\
\hline & & & & +1. & 1,2 & + & & 0,4 & $\mathrm{p}<0,01$ \\
\hline $\begin{array}{l}\text { Género Ref.: } \\
\text { HOMBRE }\end{array}$ & MUJER & & $\mathrm{nr}$ & & $\mathrm{nr}$ & $\mathrm{nr}$ & & - $\quad 0,7$ & $\mathrm{p}<0,06$ \\
\hline
\end{tabular}




\begin{tabular}{l|l|c|c|c|c}
\hline $\begin{array}{l}\text { Equipamiento } \\
\text { informático }\end{array}$ & $\begin{array}{l}\text { No poseer } \\
\text { computador }\end{array}$ & $-\quad 0,4 \mathrm{p}<0,05$ & $\mathrm{nr}$ & $\mathrm{nr}$ & \\
\hline & Poseer impresora & $\mathrm{nr}$ & $\mathrm{nr}$ & $\mathrm{nr}$ & $+2,1 \quad \mathrm{p}<0,01$ \\
\hline & Poseer escáner & $\mathrm{nr}$ & $\mathrm{nr}$ & $\mathrm{nr}$ & $+1,6 \quad \mathrm{p}<0,05$ \\
\hline
\end{tabular}

$\left.{ }^{*}\right) n r$ = "variable no retenida" por ninguna de las etapas de estimación del modelo (a $\left.p<0,15\right)$. Esto incluye las diferentes modalidades de capital cultural, condición socioeconómica, interacción entre género y condición socioeconómica, poseer o no computador, lugar de aprendizaje de la computación y edad declarada de dicho contacto.

Sentido del impacto: La modalidad de esa variable aumenta (+) o disminuye (-) la probabilidad de saber utilizar el programa indicado.

Odds Ratio promedio estimado: Una de las formas de expresar la magnitud del impacto. Corresponde a la relación entre la probabilidad de (declarar) saber utilizar el programa en vez de indicar lo contrario, comparada con la misma relación para la modalidad referencia. Ver nota 8.

Ejemplo de lectura: para el modelo correspondiente a la predicción de la variable "(declara) saber utilizar word", los estudiantes que ingresaron a la universidad con menos de 680 pts. tienen una chance de ubicarse en la situación "saben" (en vez de estar en la posición contraria), que es en promedio 0,4 veces menos importante que aquella que poseen los estudiantes de puntaje medio, a condiciones equivalentes (sexo, área de la carrera, entre otras).

Significación estadística de la relación o impacto encontrado: Una probabilidad de error de tendencia reducida implica un impacto altamente importante. Los Odds Ratio sin significación anotada presentan una probabilidad de error muy alta en el modelo ( $>0,15)$ y son considerados no significativos. En cursiva otros valores considerados. Un valor de $\mathrm{p}<0,00$ equivale a una probabilidad inferior a $0,1 \%$ de error. El valor explicativo de cada modelo es apreciado por los coeficientes $\mathrm{D}$ de Somers y el $\mathrm{R}^{2}$ Max-Resc, los que mientras más próximos a 1 indican que las variables incluidas abarcan una mayor proporción explicativa de las diferencias existentes. El Test de Adecuación de Hosmer y Lemeshow da un resultado tanto mejor que la probabilidad de error es mayor.

Tabla 5. Modelo de regresión logística (selección stepwise) para explicar la probabilidad de "saber construir páginas web" (2004; n=719).

\begin{tabular}{|c|c|c|c|c|}
\hline $\begin{array}{c}\text { Variable } \\
\text { (Mod. referencia) }\end{array}$ & Modalidades activas & \multicolumn{3}{|c|}{ Saber construir páginas web } \\
\hline & & $(1)$ & (2) & (3) \\
\hline \multirow{2}{*}{$\begin{array}{l}\text { Puntaje PSU-PAA } \\
\text { Ref. MEDIO }\end{array}$} & Bajo < 680 pts. & + & 1,3 & \\
\hline & Alto $>750$ pts. & + & 2,5 & $\mathrm{p}<0,01$ \\
\hline \multirow{6}{*}{$\begin{array}{c}\text { Área del conocimiento de la } \\
\text { carrera } \\
\text { Ref. TECNOLOGÍA }\end{array}$} & Agropecuaria & - & 0,3 & $\mathrm{p}<0,05$ \\
\hline & Artes \& Arquitectura & - & 0,3 & $\mathrm{p}<0,05$ \\
\hline & Cs. Naturales & - & 0,4 & \\
\hline & Humanidades & - & 0,2 & $\mathrm{p}<0,05$ \\
\hline & Salud & - & 0,2 & $\mathrm{p}<0,00$ \\
\hline & Cs. Sociales & - & 0,4 & $\mathrm{p}<0,05$ \\
\hline $\begin{array}{c}\text { Género } \\
\text { Ref. HOMBRE }\end{array}$ & MUJER & - & 0,6 & $\mathrm{p}<0,05$ \\
\hline
\end{tabular}




\begin{tabular}{c|l|c|c|c}
\hline $\begin{array}{c}\text { Capital cultural (familiar) } \\
\text { Ref. ALTO }\end{array}$ & Bajo & + & 1,5 & \\
\cline { 2 - 5 } & Medio & - & 0,5 & $\mathrm{p}<0,02$ \\
\hline \multicolumn{2}{c|}{ Años de experiencia } & + & 1,2 & $\mathrm{p}<0,01$ \\
\hline \multirow{2}{*}{$\begin{array}{c}\text { Dominio de internet } \\
\text { Ref. MEDIO }\end{array}$} & Bajo & - & 0,4 & \\
\cline { 2 - 5 } & Alto & + & 3,6 & $\mathrm{p}<0,00$ \\
\hline \multicolumn{2}{c|}{ Uso total de internet (ver cuadro No 2) } & & $14,2 \%$ & $\mathrm{p}<0,01$ \\
\hline \multicolumn{2}{c}{ Probabilidad observada } & & 0,67 & \\
\hline \multirow{2}{*}{$\begin{array}{c}\text { Indicadores de calidad } \\
\text { del modelo }\end{array}$} & D de Somers & & 0,31 & \\
\cline { 2 - 5 } & $\mathrm{R}^{2}$ Max-Rescaled & & $\mathrm{P}<0,72$ & \\
\cline { 2 - 5 } & Test de Adecuación H\&L & & & \\
\hline
\end{tabular}

En este caso el análisis incluyó las variables "años de experiencia", "actitudes", "lugar de aprendizaje", "lugar de acceso", "dominio de" y "uso total" de internet. Una vez más, sólo las variables con impactos significativos fueron retenidas por el modelo. Para el caso de variables continuas la interpretación es la misma (sentido del impacto y significación). Cada año suplementario de experiencia en internet multiplica por 1,203 (Odds Ratio promedio para esa variable según el modelo) las posibilidades de "saber hacer páginas web". En otras palabras, a condiciones comparables, el solo hecho de poseer un año más de experiencia aumenta en promedio en un 20\% dicha probabilidad (en relación con su contrario: "no saber").

Ambas tablas exponen las variables más asociadas a la utilización y conocimiento de ciertos programas de uso corriente. La inclusión de la variable "área del conocimiento de la carrera" responde a la hipótesis previa de que la orientación vocacional de los estudiantes y las exigencias que ello supone se ligan a la disposición de uso de ciertos programas, lo que se confirma con los resultados expuestos. No obstante, es importante considerar que en estos casos no contamos con información que permita controlar los incentivos o exigencias de uso de programas que, por ejemplo, puedan realizar ciertos docentes o asignaturas. Ello invita a tomar con cautela estos resultados y considerarlos más bien como una radiografía de la situación por área del conocimiento.

Mientras el procesador de textos Word no presenta usos diferenciados según área, para el caso de las planillas de cálculo Excel los estudiantes de áreas como "Humanidades" o "ArtesArquitectura" tienden a conocerles significativamente menos que los estudiantes del área "Tecnología". De manera general, aquellos del área "Humanidades", en condiciones comparables, tienden a utilizar menos programas que el resto de los estudiantes, lo que podría ser materia de atención especial. 
Globalmente, los tres softwares de uso corriente son dominados por la mayoría de los estudiantes (>60\%) y, hecho importante, no aparecen usos diferenciados entre hombres y mujeres. En total, tres estudiantes de cada diez se inscriben en el primer caso, y sólo uno sobre siete en el segundo. En este último, el predominio del área "Tecnología" (donde el 33\% declara "saber") resulta significativo respecto de las otras áreas, salvo para "Ciencias. Naturales". Tanto para esta variable como para el manejo de "otros programas", las mujeres se ausentan significativamente más que los hombres de dichas categorías.

En otro plano, resulta interesante ver la diferenciación del "saber hacer páginas web" entre estudiantes de capital cultural familiar medio respecto de aquellos más aventajados. En condiciones equivalentes, éstos presentan 0,5 veces menos de chances de "saber hacer páginas web" (en lugar de no saber) que los estudiantes con alto capital cultural familiar. Lo mismo ocurre respecto del capital escolar en el cual los estudiantes más aventajados en su ingreso a la universidad exhiben 1,4 veces más de posibilidades de declarar poseer dicho dominio que sus homólogos con capital escolar medio. Otro tanto ocurre cuando se observa la relación positiva entre equipamiento y dominio de "otros programas". Finalmente, como se indica en la lectura de la tabla 5, se incluyeron en tanto variables control factores como la "experiencia", el "dominio" y "uso total" de internet, los que, sin sorpresa, aparecen significativa y positivamente ligados a la capacidad de hacer páginas web.

Estos resultados insisten en la relación entre ciertas disposiciones y los capitales culturales materiales a los cuales se tiene o se ha tenido acceso, y que facilitan y/o promueven una apropiación más profunda de tales herramientas. En otras palabras, esta relación se convierte en una clave de dominio superior que contrasta con lo que parece ser una apropiación más funcional, por ejemplo, considerando el uso "música..." (tabla 3) que exhiben quienes poseen un background cultural más limitado. Estos resultados interpelan la problemática de la apropiación de las herramientas tecnológicas por parte de la población 
en sus aspectos más sutiles pero, a la vez, más fundamentales y determinantes.

Notemos finalmente que la variable socioeconómica no aparece directamente presente en ningún sentido ni modalidad.

\section{Efectos indirectos de las diferencias socioeconómicas}

En primer lugar, a partir de la evidencia empírica construida los resultados deben ser vistos como el producto de un análisis situado y referido a un momento ${ }^{14}$. La primera invitación es a considerarlos con prudencia, sobre todo ante los eventuales cambios operados a partir de $2004^{15}$.

Más allá de lo anterior, pese a la homogeneidad esperable de una población universitaria producto de múltiples selecciones previas, sociales y escolares, y considerando que se trata de una población $100 \%$ conectada y usuaria, los análisis permiten afirmar que existen signos claros de la existencia de brecha digital entre individuos socioeconómicamente diferentes. Ello de manera previa a la exigencia que implica el programa CFG.

Esta brecha digital sería el producto de diferencias significativas en el acceso y la tenencia de herramientas informáticas. De esa manera, son los capitales culturales materiales quienes explican prioritariamente las diferencias de comportamientos evidenciados (horas de conexión, tipos de usos). Al mismo tiempo, no es posible afirmar que existan diferencias constantes de capitales culturales incorporados en términos socioeconómicos, principalmente respecto de internet. Una de las hipótesis de análisis se ve refutada, pues todo

14 Los sesgos propios de la muestra son vistos, en este caso, como elementos positivos y no parecen ameritar métodos de corrección por selección. Por su parte, el conjunto de las variables continuas presenta distribuciones normales.

15 En particular, tres aspectos merecerían ser revisados después de 2004: el acceso a ordenadores portátiles y a pen drive y sus niveles de impacto (equipamiento); la eventual ampliación de cobertura de la banda ancha o similares (acceso), y los comportamientos respecto de los blogs (usos). Ver: http://www.wipchile.cl/estudios/WIP_Chile_2006 informe.pdf 
parece indicar que, salvadas las diferencias materiales, la variable socioeconómica no determinaría en lo grueso las disposiciones, apreciaciones y usos frente a las NTIC.

En otras palabras estamos aquí en presencia de efectos indirectos de la condición socioeconómica. No obstante, es posible preguntarse si tales impactos podrían ser o más fuertes o expresarse de manera directa al considerar otros grupos en los cuales la llamada exclusión digital (Raad, 2006) pueda estar más presente.

Por otro lado, las diferencias de comportamiento y de dominio se encuentran generalmente ligadas a capitales culturales familiares y escolares, los que, a su vez, se relacionan con la condición socioeconómica de los estudiantes. Se aprecia además el carácter multiplicativo y sinérgico entre tipos de capitales. Un mayor equipamiento tiende a potenciar mejores disposiciones, usos más integrales y cotidianos, y mejores destrezas y autopercepciones. Otro punto importante es la dicotomía evidenciada entre aproximaciones a internet -integrales (de apropiación), versus funcionales (limitadas)-, el género (en interacción con la condición socioeconómica) y diferencias en términos de acceso y de background cultural familiar.

\section{Las principales diferencias son de género}

La principal y más persistente variable respecto de la brecha digital analizada es el género. Las mujeres muestran, en condiciones comparables, comportamientos y disposiciones diferentes a los hombres en tiempo, frecuencias y tipo de usos, en dominio de herramientas y autopercepción. Esta constatación resulta coherente con estudios internacionales ${ }^{16}$. No obstante, ciertos países desarrollados exhiben niveles similares para ambos sexos en lo que respecta a

16 Por ejemplo, PISA-2003 constataba que un 99,3\% de jóvenes europeos de 15 años declaraba haber ya utilizado un computador y que $81 \%$ posee uno en su casa. No obstante, los hombres les utilizan con mayor frecuencia y desde hace más tiempo, y aunque no existen diferencias para usos como tratamiento de textos o comunicación, sí las hay para juegos y programación (EURIDYCE, 2005). Ver también el Widening Women's Work in Information and Communication Technology (http://www.ftu-namur.org/www-ict). 
conexión, sobre todo cuando los niveles de democratización del acceso son elevados (Bernier et al., 2005), tendencia que debemos explorar en el caso chileno.

Indudablemente, la socialización diferenciada entre hombres y mujeres, que determina representaciones, aspiraciones y prácticas distintas según género, parece hacerse presente con fuerza en los usos y apropiación de las NTIC. Nuestros resultados invitan a profundizar los análisis locales respecto de la socialización general y escolar de las mujeres. Si bien la tendencia en los países occidentales es que a mayor desarrollo social y económico las aspiraciones de las familias en torno a la escolaridad tienden a ser equivalentes para ambos sexos, en la práctica subsisten una serie de mecanismos sutiles pero acumulativos que diferencian desde temprana edad la autopercepción y los comportamientos de niños y niñas (Duru-Bellat, 2004). Es posible postular que a las diferencias que la literatura anota sobre lecturas, gustos, niveles de autonomía escolar y de control familiar, y orientación de los estudios, se agregan también las referidas a la apropiación de las NTIC. Al mismo tiempo, éstas pueden cobijar y reproducir las anteriores.

Más allá del rol del sistema educativo, una hipótesis correlaciona los usos y comportamientos referidos a internet a la relación y apropiación -más limitada- que las mujeres establecen desde temprana edad con el espacio físico y geográfico (Duru-Bellat, 2004). Otra veta, no contradictoria con las anteriores, es la de los imaginarios tecnológicos (matemático, lúdico, moderno, por ejemplo) y las relaciones sociales implicadas en el uso de herramientas.

Notemos además que la literatura muestra, por un lado, que las diferencias de socialización por género tenderían a ser más fuertes en las familias en desventaja socioeconómica y, por otro, que las prácticas de las familias respecto de la inversión educativa tienden en algunos casos a ser menos acentuadas en términos relativos cuando se trata de la educación de las mujeres (Duru-Bellat, 2004). Ello podría ser consistente, por ejemplo, con no poseer conexión banda ancha ni 
computador individual (gráfico 1), condición esta última que aparece fuertemente ligada al género en condiciones controladas. Aunque nuestros análisis no siempre muestren efectos de interacción entre la condición socioeconómica y el género, resulta evidente que las mujeres de escasos recursos -incluso las que llegan a la universidadconstituyen un segmento que acumula numerosas desventajas.

\section{Estrategias país y profundización de los análisis en la educación superior}

Respecto de las estrategias y enfoques para el desarrollo tecnológico del país, una parte importante de los resultados aquí encontrados ponen de relieve que, incluso en un segmento de alto capital humano, existen diferencias que invitan a ir más allá de los problemas referidos al equipamiento. La experiencia chilena estaría necesitando no sólo considerar los indicadores materiales para poner atención en los procesos ligados a las NTIC (Araya, 2005). Al mismo tiempo, las perspectivas de desarrollo del país dependerían de la superación de la dificultad para "asegurar la transición tecnológica de recursos humanos y organizativa a un modelo informacional de desarrollo" (Castell, 2003). Los resultados aquí expuestos, aunque localizados y modestos, parecen respaldar ambas proposiciones.

Siempre podrá argumentarse que existe un umbral de tolerancia para la brecha referida a usos y a dominios, en la medida en que ella no interfiera ni perjudique adicionalmente las actividades fundamentales de la vida de los individuos, en este caso los estudios. También pueden aparecer tendencias compensatorias -como lo insinúan algunos resultados (tabla 3) - que privilegien el "uso para el estudio" cuando ello se hace indispensable, limitando así el impacto de las diferencias aquí vistas.

Sin embargo, aun cuando los efectos de la incorporación de NTIC a la enseñanza puedan ser socialmente neutros, nuestros análisis nos invitan a pensar que, aparejados a esta brecha, coexisten una serie de otras variables ligadas a los desempeños y logros académicos, y 
que pueden convertirse en factores que acentúen las desigualdades en educación, de no ser adecuadamente considerados.

Desde el punto de vista de la responsabilidad de las instituciones universitarias, son indispensables e irreemplazables los esfuerzos dirigidos a ofrecer condiciones equitativas de acceso, de formación y de dominio de herramientas, so pena de acentuar brechas que serían, además, nocivas para la elevación de la calidad en el sistema.

Considerando la evidencia respecto de las diferencias en términos de capitales escolares y especialmente de prácticas de estudio -por ejemplo, en los estudiantes de primer año (Roco, 2006)-, la incorporación de NTIC en tanto herramientas para el estudio requiere de contextualización, explicitación y de más y mejores métodos y oportunidades de aprendizaje. La imagen de un estudiante abrumado en la biblioteca a la que entra por primera vez no cambia esencialmente cuando reemplazamos fichas y estanterías por un mouse, una pantalla y un cable a 20MB/s. Nuestros análisis invitan a pensar el estatus de las NTIC como herramienta de estudio en un nivel no tan diferente de otras herramientas, aparentemente más clásicas (bibliotecas y guías, por ejemplo).

Que los estudiantes puedan y sepan servirse de ciertos útiles incorporándolos adecuadamente a sus prácticas de estudio requiere de una mayor atención si se pretende disminuir el fracaso y la deserción. Las NTIC proporcionan una buena oportunidad para ello si se propone seriamente y se realiza eficazmente. Como insiste el sociólogo Rubén Araya, toda tecnología es siempre un intento por asistir o modificar actividades y prácticas sociales. El punto de fondo es que la incorporación de NTIC al sistema educativo no implique ni la manutención ni la ampliación de las barreras de discriminación social ya existentes (capitales culturales), como tampoco la acentuación o creación de otras (capitales escolares y competencias cognitivas y metacognitivas).

Aparte de la equidad en el acceso, resulta necesario poner atención en: i) prácticas de estudio, nivelación de capitales 
escolares, desarrollo de habilidades generales, manejo y selección de información, toma de apuntes, por ejemplo (Romainville et al., 2003; Romainville, 2000), y ii), la calidad y eficacia de las prácticas pedagógicas. Es fundamental y estratégico que el uso de las NTIC se vea contextualizando en ese marco y no al revés (Leclercq, 1997; Lebrun, 2002).

La experiencia de los CFG de la Universidad de Chile abre importantes vetas de exploración. A la luz de la relativa decepción respecto de la tan anunciada revolución de la enseñanza terciaria gracias a las NTIC (OCDE, 2005), se hace indispensable que su creciente incorporación a los cursos universitarios se haga con resguardo de las diferencias aquí consideradas, pero, al mismo tiempo, a partir de un rediseño del proceso de enseñanza-aprendizaje y de las prácticas pedagógicas. Ese rediseño debe, por cierto, cautelar la adecuada evolución conjunta de las dimensiones de equidad y calidad.

Agradecemos a Roxana Pey y Sara Chauriye de la Unidad de Formación Básica y General y a María Eugenia Horvitz de la Dirección de Bienestar Estudiantil de la Universidad de Chile por su colaboración en la recolección de los datos que sirven de base al presente artículo.

\section{Referencias bibliográficas}

Araya, R. (2005) Chile necesita pasar a una segunda generación de politicas públicas sobre TIC.

http://www.ticschile.cl/documentos/entrevista_raraya.pdf Último acceso el 21/01/07.

Brunner, J. J. (2001) Chile: Informe sobre capacidad tecnológica. Santiago de Chile: PNUD, pp. 22-29

Bernier, Ch. \& Laflamme, S. (2005) Femmes, hommes et usages d'internet: ségrégation ou différenciation? Canada; Université Laurentienne, pp. 917. XVIle Congrès International des Sociologues de langue française; Tours, 5-9 juillet 2004 http://w3.univ-tlse2.fr/aislf/gtsc/DOCS_SOCIO/FINITO_PDF/ BernierLaflamme.pdf Último acceso el 21/05/07. 
Bourdieu, P. (1979) Les trois états du capital culturel. Actes de la recherche en sciences sociales, (30), pp. 3-6.

Castell, M. (2003) El Modelo Democrático Chileno de Desarrollo. Foro Bicentenario 2003: Chile Hoy iAcercándonos al umbral del desarrollo? Santiago de Chile: Comisión Bicentenario, pp.104-107.

CERI - OCDE. E-Learning in Tertiary Education: Where do we stand? (2005). Paris: OCDE, pp. 219-224.

Duru-Bellat M. (2004) L'école des filles. Quelle formation pour quels rôles sociaux? Paris: L'Harmattan, pp. 83-135

EURIDICY. (2005) How boys and girls in Europe are finding their way with information and communication technology. http://www.eurydice. org/ressources/eurydice/pdf/0_integral/069EN.pdf Último acceso el 21/05/07.

Godoy, S. \& Herrera, S. (2004) Internet usage in Chile and the World: first results of the World Internet Project-Chile, pp. 1-17

http://www.wipchile.cl/estudios/japan/WIP_article_1_CdINFO_for_ Japan_jul04.pdf Último acceso el 21/05/07.

Hopenhayn, M. (2003) Educando para la sociedad del conocimiento: logros alcanzados y desafíos para el futro. Foro Bicentenario 2003: Chile Hoy ¿Acercándonos al umbral del desarrollo? Santiago de Chile: Comisión Bicentenario, p. 71.

Jaramillo, O. y Castellón, L. (2001) Las múltiples dimensiones de la brecha digital en Chile. Reflexiones Académicas, No 15 Santiago de Chile: UDP, pp.81-100.

Jaramillo, O. y Castellón, L. (2002) Tele-centros, Usuarios y apropiación de las TICs. Santiago de Chile: UDP

Leclercq, D. (1997). Trois orbites de réflexion en pédagogie universitaire. In: Boxus et al. Stratégies et médias pédagogiques pour l'apprentissage et l'évaluation dans l'enseignement supérieur. Actes du XV Congrès de l'Association Internationale de Pédagogie Universitaire (AIPU).

Lebrun, M. (2002). Des technologies pour enseigner et apprendre. Bruxelles: De Boeck Université, pp.1-240.

PNUD. (2002) Desarrollo humano en Chile: nosotros los chilenos: un desafío cultural. Santiago de Chile: PNUD, pp.297-348

Raad, A.M. (2006) Exclusión digital: nuevas caras de viejos malestares. Revista 
MAD (14). http://www.revistamad.uchile.cl/14/raad.pdf Último acceso el 21/05/07.

Roco, R. (2006) Évaluation de l'équité et de l'efficacité de la composante web des Cours de Formation Générale (CFG) à l'Université du Chili. MonastirTúnez: Actes du XXIII Congrès de l'AIPU. 15-18 Mai.

Romainville, M. (2000) L'échec dans l'Université de masse. Paris: L'Harmattan, pp. 55-108.

Romainville, M. et Noël, B. (2003) Métacognition et apprentissage de la prise de notes à l'université.Arob@se, Vol 1-2.pp. 87-96. www.arobase.to Último acceso el 21/05/07.

Soupizet, J-F. (2003) La Fracture Numérique: mesures et spécificités. Actes $\mathrm{du}$ Colloque International : Les fractures numériques Nord/Sud en question: Quels enjeux? Quels partenariats?

Gironde-France 25-28 août. pp.1-20

http://www.africanti.org/resultats/colloque2003/Communications/ SOUPIZET1.pdf Último acceso el 21/05/07.

Recibido: 30 de marzo de 2007

Aceptado: 30 de abril de 2007 